\title{
Brechas, representación y congruencia élite-ciudadanía en Chile y Uruguay
}

\author{
Gaps, Representation, and Elite-Mass Congruence \\ in Chile and Uruguay
}

\author{
Bastián González-Bustamante (D) http://orcid.org/0000-0003-1510-6820 \\ Universidad de Santiago de Chile, Chile, bastian.gonzalez.b@usach.cl
}

Recepción:

08/08/2018

Aprobación:

$26 / 10 / 2018$

\begin{abstract}
This paper analyzes three dimensions of elite-mass congruence in Chile and Uruguay: (a) ideological; (b) public issues, support, and satisfaction with democracy; and, (c) economic preferences on the role of the State. Data from probabilistic national surveys and surveys on elites financed by IDRC-Canada and carried out from 2013 to 2014 in each country are used. Congruence indexes are calculated to identify gaps between citizens and representatives. The main indexes used are Relative Citizen Congruence and Earth Mover's Distance, a recent innovation borrowed from computer science. The findings show a greater ideological congruence in Chile, a greater congruence on support to democracy in Uruguay, and important gaps on education issues in Chile. Furthermore, in both countries there is greater congruence regarding relevant public issues between higher socioeconomic level population and the political elite.
\end{abstract}

Key words: elites, representation, congruence, Chile, Uruguay.

Resumen: Este artículo analiza tres dimensiones de la congruencia éliteciudadanía en Chile y Uruguay: a) ideológica; b) temática, respaldo y satisfacción con la democracia; y c) preferencias del rol del Estado en la economía. Se utilizan datos de encuestas nacionales probabilísticas y encuestas de élites financiadas por IDRC de Canadá y realizadas entre 2013 y 2014 en cada país. Se calculan indicadores de congruencia para identificar brechas entre ciudadanos y representantes. Los principales indicadores con los cuales se trabaja son Congruencia Ciudadana Relativa y Earth Mover's Distance, una innovación reciente de las ciencias de la computación. Los hallazgos muestran una mayor congruencia ideológica en Chile, una mayor congruencia en el apoyo a la democracia en Uruguay e importantes brechas en materia educacional en Chile. Además, en ambos países se advierte mayor congruencia sobre problemas públicos relevantes entre los sectores de mayor nivel socioeconómico y la élite política.

Palabras clave: élites, representación, congruencia, Chile, Uruguay. 


\section{Introducción $^{1}$}

En las democracias contemporáneas, los sistemas políticos poseen procesos que permiten agregar las preferencias y canalizar las demandas ciudadanas. Estos procesos se vinculan con la representación política y los mecanismos de sufragio, los cuales, por su parte, operacionalizan la elección de autoridades y, a su vez, generan una separación entre aquellos que gobiernan y quienes son gobernados. Esto suele asociarse con la existencia de ciertos supuestos como, por ejemplo, que los ciudadanos eligen representantes alineados con sus preferencias o, por otro lado, que la élite política tiene incentivos para canalizar adecuadamente las demandas ciudadanas para obtener apoyo popular, mantener su legitimidad y perpetuarse en el poder.

Estos supuestos se alinean con la teoría electoral de la representación y con una confluencia de la responsabilidad y el mandato en el marco de la teoría de la representación. Mientras la teoría electoral asume que la ciudadanía elige libre y periódicamente a sus representantes, por otro lado, comprender la representación como una confluencia entre responsabilidad y mandato implica suponer que la élite responde antes sus gobernados y que, además, son sus agentes o delegados (Pasquino, 2011; Sartori, 1999). Sin embargo, esto no necesariamente puede tener un correlato empírico. De hecho, la teoría consensual de la representación asume que la ciudadanía consiente las decisiones de los gobernantes (Sartori, 1999), lo cual tiende a alejar a la representación de concepciones participacionistas.

En este contexto, preguntarse sobre qué tan cerca están las preferencias de la élite política con las de los ciudadanos implica evaluar las brechas que existen entre gobernantes y gobernados en el sistema político. El trabajo clásico de Miller y Stokes (1963) puede identificarse como un punto desde el cual se abrió una línea de investigación en torno a las preferencias de los ciudadanos y sus representados. La congruencia política puede, en este contexto, entenderse como el grado de correspondencia entre las preferencias de la ciudadanía y las posiciones de sus representantes (Powell, 2004; RealDato, 2017).

1 Este artículo fue financiado por IDRC de Canadá a través del proyecto "A Crisis of Legitimacy: Challenges of the Political Order in Argentina, Chile, and Uruguay" (Alfredo Joignant, investigador responsable). Los resultados preliminares de esta investigación fueron presentados en el XXXIV Congreso Internacional de la Asociación de Estudios Latinoamericanos, posteriormente: LASA, Nueva York, mayo de 2016. Agradezco el apoyo financiero de LASA (Travel Grant 2016/40071). También agradezco los comentarios de Antoine Maillet y las recomendaciones de Lucía Selios. 
Esto resulta relevante por los posibles vínculos con la teoría de la representación y de la democracia; ${ }^{2}$ sin embargo, también tiene una importancia práctica debido a la preocupación creciente por la existencia de brechas entre la élite y la ciudadanía, marco en el cual una eventual ruptura de la relación principal-agente puede asociarse con una crisis de representatividad (Mainwaring, 2006).

En este sentido, es importante aclarar que estas conceptualizaciones tienen foco en la representatividad; por lo tanto, más que ciudadanía, como concepto amplio, se hace referencia a los votantes específicamente, quienes se pueden entender como aquel segmento de la ciudadanía que posee derechos políticos. ${ }^{3}$

Este trabajo describe los niveles de congruencia élite-ciudadanía en Chile y Uruguay con métodos de medición que permiten una desagregación en dimensiones enfocadas en la autoidentificación ideológica, temas de políticas públicas, apoyo y satisfacción con la democracia, y preferencias sobre el rol del Estado en la economía. Se utilizan datos de encuestas nacionales y encuestas aplicadas a la élite política de ambos países entre 2013 y 2014 , para determinar si las percepciones de los gobernantes se alinean con las de la ciudadanía en un nivel agregado. Además, se realizan distinciones dependiendo del nivel socioeconómico de la población chilena y uruguaya, lo cual permite identificar la existencia de mayores alineamientos entre gobernantes e individuos con mayores recursos sociales y económicos, lo que podría ser un rasgo de desigualdad política.

Los países de este estudio son, junto con Argentina, parte del Cono Sur de América Latina. Tanto Chile como Uruguay tienen presidencialismos con altos niveles de desarrollo humano en la región. Por otra parte, si bien los dos países tienen un alto nivel de calidad de la democracia (Levine y Molina, 2011), poseen diferencias actitudinales relevantes. Mientras Chile tiene altas tasas de desafección política y baja confianza en las instituciones (Siavelis, 2017), Uruguay es líder a nivel regional en estos aspectos (Morales, 2014).

2 Es importante indicar, tal como señala Otero-Felipe (2014), que la congruencia es un indicador de que existe representación política en sus dimensiones sustancial o representativa, de acuerdo con la tipología de Pitkin (1967). Sin embargo, en estricto rigor, congruencia y representación política no son términos equivalentes.

3 En este contexto, es esencial indicar que hay distintas dimensiones asociadas al concepto ciudadanía. Marshall (1950), tempranamente, distinguía entre ciudadanía civil, política y social. En general, estos tres tipos de ciudadanía suelen operar de forma separada, y solo la intersección de estas tres categorías implicaría una ciudadanía democrática plena (PNUD, 2014). 
Este artículo se divide en cuatro apartados. Primero, se presenta un apartado teórico que ofrece un breve estado del arte sobre los estudios de élites y la medición de congruencia élite-ciudadanía. Segundo, se desarrolla un apartado metodológico donde se detallan las encuestas utilizadas, la operacionalización de variables y los índices de congruencia usados para medir tres dimensiones: a) congruencia ideológica por bloques políticos; b) congruencia temática, apoyo y satisfacción con la democracia; y c) congruencia en preferencias sobre el rol del Estado en la economía.

Luego, se muestran los resultados de las tres dimensiones de análisis. Finalmente, se exponen las conclusiones con un resumen de los principales hallazgos.

\section{Revisión de literatura}

\section{Estudios de élites y brechas élite-ciudadanía}

Si bien la élite política y los gobernantes como objeto de estudio preceden al surgimiento del Estado moderno, un punto de partida relativamente contemporáneo y aceptado por la literatura especializada se puede fijar con el auge teórico del elitismo clásico a fines de siglo XIX. Los principales exponentes del elitismo clásico fueron Mosca, Pareto y Michels. Décadas después, a mediados del siglo XX, se advierte una división en el estudio de las élites y es posible identificar un enfoque más bien pluralista y, por otra parte, uno de corte unitario (González-Bustamante, 2016; Joignant, 2011).

En el marco del enfoque pluralista es posible situar trabajos como Dahl (1961), el cual asume la existencia de una dispersión del poder, ya que diversos grupos pugnan por las posiciones dominantes. En oposición a esto, Mills (1956), uno de los exponentes más relevantes del enfoque unitario, desarrolla la idea de que no existe una relación de equilibrio en la sociedad, pues la élite política está compuesta por individuos que se ubican en la cúspide social, posición que no obtienen necesariamente por sus capacidades personales, ya que en el proceso median fuentes de riqueza y el mecanismo de la celebridad.

En este escenario, durante la segunda mitad del siglo XX, la sociología de Pierre Bourdieu emergió como una aproximación teórica útil para los estudios empíricos sobre élites. Su trabajo teórico encuadra una conceptualización sobre el funcionamiento del espacio social, los campos de poder, los recursos y capitales de los individuos, además de las estructuras estructurantes (habitus) (González-Bustamante y Garrido-Vergara, 2018; Serna, 2006). Los recursos y capitales de un individuo se asocian con un lenguaje 
de competencias y, en consecuencia, a desigualdades: el acceso al poder y la capacidad de conservarlo se da en un contexto de naturalización de atributos individuales, que en estricto rigor se vinculan con una herencia de capitales (Alcántara, 2013; González-Bustamante, 2016; Joignant, 2011).

Lo anterior implica que el principio de igualdad de la representatividad democrática está asociado con profundas desigualdades. Esto hace que, desde la teoría de las élites, el principio de igualdad en las democracias contemporáneas sea más bien una aspiración y no necesariamente una realidad (Arana, 2016; Parry, 2005). Además, el predominio de la representación electoral en las democracias modernas también se asocia con desigualdades.

Un supuesto teórico persistente durante el último medio siglo ha sido la existencia de un sesgo de clase en la participación política (Lipset, 1960). En este sentido, actualmente tanto la participación electoral como las formas no convencionales de participación política de corte contencioso, que a comienzos del siglo XX eran propias de sectores marginados, tienden a fortalecer la opinión de los individuos con más recursos sociales y económicos, cristalizando, de esta forma, una desigualdad política (Dalton et al., 2010; Dubrow, 2008).

En este contexto, durante la mayor parte del siglo XX, la teoría de élites ha buscado responder a la pregunta: quién gobierna. Para esto ha esbozado, con distintos matices, una respuesta similar: una pequeña minoría organizada (Bobbio, 2003). Así, durante las décadas recientes, los estudios sobre élites políticas se han concentrado en sus características, su rol en procesos políticos como las transiciones democráticas, su nivel de cohesión y su congruencia con la ciudadanía. ${ }^{4}$

Sobre consenso y congruencia hay trabajos que se han enfocado en las brechas ideológicas entre la élite y la ciudadanía. En esta línea es posible identificar, por una parte, trabajos cuyo foco es la congruencia temática (Alcántara y Luna, 2004; Kitschelt, 2000; Luna y Zechmeister, 2005). Por ejemplo, Luna y Zechmeister (2005) encuentran mayores niveles de congruencia élite-ciudadanía en los sistemas de partidos más consolidados y en los partidos más de izquierda en América Latina. Por otra parte, se pueden hallar trabajos cuyo foco es la congruencia entre las preferencias de los representantes y representados, como, por ejemplo, Andeweg y Thomassen (2005) o Barreda y Ruiz (2015).

4 Véase, por ejemplo, Best (2011), Gulbrandsen y Engelstad (2005), Higley y Gunther (1992) y Pakulski (2012). Para estudios recientes en América Latina y el Cono Sur, véase Codato y Espinoza (2018), González-Bustamante y Olivares (2018) y Vommaro y Gené (2018). 
La congruencia entre representantes y representados favorece la atención a las demandas ciudadanas (responsiveness), lo que a su vez beneficia la calidad de la representación y de la democracia (Barreda y Ruiz, 2015). Un gobierno es responsivo cuando impulsa políticas y decisiones vinculadas con los intereses del electorado.

En este sentido, la alineación entre representación y responsiveness se da en contextos donde el electorado cuenta con altos grados de información; además, puede considerarse una medida de cuanto accountability permite la estructura institucional (Przeworski et al., 1999). De no producirse esta alineación se generan brechas entre votantes y representantes, las cuales pueden generar corrientes de desafección, las que podrían ser un predictor de colapsos en los sistemas de partidos, realineamientos electorales o crisis de representación (Morales, 2014).

En este contexto, si se considera a las crisis de representación, siguiendo a Mainwaring (2006), como una ruptura en el continuum principal-agente que se plasma en la relación élite-ciudadanía, entonces la intermediación política dejaría de funcionar adecuadamente. Esto puede tener distintas consecuencias: disminución en la participación electoral, aumento de la movilización social, surgimiento de outsiders o aparición de movimientos antisistema. ${ }^{5}$

Previo a una crisis de representatividad, se puede identificar lo que Joignant et al. (2017) han denominado malestar. Este concepto combina la desconfianza en las instituciones, la desaprobación a la gestión gubernamental y la desafección política (Siavelis, 2017). Dichas dimensiones pueden variar en una serie de tiempo y si bien no necesariamente constituyen predictores de crisis, su presencia es una antesala a la ruptura del continuo de representatividad (Cantillana et al., 2017).

Por otra parte, el estudio de la congruencia también permite conocer mejor los vínculos entre representantes y representados (Barreda y Ruiz, 2015). En este sentido, si se enfoca en los partidos y sus votantes, se puede asociar a los vínculos programáticos de representación (Dalton, 2011; Selios, 2015). Por ejemplo, los vínculos programáticos tienden a ser más fuertes en las democracias donde los electores tienen preferencias similares a las de los representantes, situación que tiende a condicionar la disposición y capacidad para cumplir los programas de gobierno, ya que de no ocurrir esto los votantes dejarían de apoyar a los partidos (Kitschelt et al., 2010; Luna y Zechmeister, 2005 y 2010; Morales, 2014).

5 Esta ruptura se puede explicar por factores económicos (Bellinger y Arce, 2011) o por la ausencia de capacidad institucional (Kitschelt et al., 2010; Torcal, 2006). 


\section{Medición de congruencia élite-ciudadania}

La congruencia élite-ciudadanía, como se indicó en el apartado anterior, tiende a favorecer la estabilidad democrática. Por el contrario, la existencia de brechas se asocia con crisis de representatividad y problemas en el sistema político y en el de partidos. Vista la relevancia de la congruencia, es necesario reflexionar respecto a su medición.

Una definición operacional de congruencia se vincula directamente con el grado de coincidencia entre representantes y representados, lo que se ha medido con diferentes técnicas y formas de operacionalización (Rivas et al., 2014). Utilizar distancias entre medias o promedios y distancias simples, con base en estimaciones puntuales, ha sido un método clásico y bastante sencillo; sin embargo, presenta problemas metodológicos asociados con imprecisiones en la medición (Golder y Stramski, 2010; Otero-Felipe, 2014).

De forma similar, la correlación de actitudes, al no considerar la distribución estadística de las opiniones, tiende a generar sobreestimaciones y subestimaciones en la medición (Achen, 1977; Weissberg, 1978). ${ }^{6}$

En los últimos años, en el contexto de la búsqueda por superar las ineficiencias asociadas con los métodos de medición menos sofisticados, se ha popularizado el trabajo de Golder y Stramski (2010), que distingue tres tipos de congruencia: a) one-to-one, entre ciudadanos y representantes, la cual puede ser alta cuando la distancia absoluta entre los ciudadanos y representantes es pequeña; b) many-to-one, enfocada en la congruencia entre varios ciudadanos y un representante; y c) many-to-many, que busca medir la congruencia entre varios ciudadanos y varios representantes. Siguiendo los lineamientos metodológicos de Golder y Stramski (2010), se puede medir la congruencia manyto-one con la Congruencia Ciudadana Absoluta Mediana (Absolute Median Citizen Congruence, posteriormente: AMCC) (i), donde $M C$ es el posicionamiento ideológico promedio de la ciudadanía y $G$, del gobierno o de la élite.

(i) $\mathrm{AMCC}=|M C-G|$

6 En este contexto, los indicadores de proximidad, centrismo y receptividad (responsiveness) de Achen (1978) fueron, en su época, medidas más precisas que las distancias entre medias o estimaciones puntuales. 
También es posible medir la Congruencia Ciudadana Absoluta (Absolute Citizen Congruence, posteriormente: ACC) (ii), medición que incorpora cierta información sobre la distribución de las preferencias ciudadanas y permite obtener mayor precisión. En la fórmula, $N$ indica el número de ciudadanos, y $C_{i}$ es la identificación del $i^{\text {th }}$ ciudadano.

$$
\text { (ii) } \mathrm{ACC}=\frac{1}{\mathrm{~N}} \sum_{i=1}^{\mathrm{N}}\left|\mathrm{C}_{i}-\mathrm{G}\right|
$$

Por último, otra posibilidad es la Congruencia Ciudadana Relativa (Relative Citizen Congruence, posteriormente: RCC) (iii), que contiene información útil sobre la congruencia entre los ciudadanos y sus representantes, al incorporar la dispersión de las preferencias ciudadanas. Esta medición va de cero a uno, mientras más congruencia existe, el indicador es más cercano a cero. De forma similar, cuando los valores de AMCC y ACC son bajos, hay mayor congruencia.

$$
\text { (iii) RCC }=1-\frac{\sum_{i=1}^{N}\left|C_{i}-M C\right|}{\sum_{i=1}^{N}\left|C_{i}-G\right|}
$$

Por otra parte, la congruencia many-to-many evalúa cuán similares son las distribuciones de las preferencias, donde $F_{1}(\mathrm{x})$ y $F_{2}(\mathrm{x})$ son funciones de distribución acumulativa (Cumulative Distribution Functions, posteriormente: CDF) de las preferencias de la ciudadanía y de la élite, respectivamente; esta medición también es conocida como Manhattan (iv).

$$
\text { (iv) Manhattan }=\Sigma_{x}\left|F_{1}(X)-F_{2}(X)\right|
$$

Cuando la distribución de las preferencias es idéntica, el área entre cada CDF es cero y la congruencia es perfecta (Golder y Stramski, 2010). En este sentido, si se considera una distancia $Ð$ como una descripción numérica de cuán alejados están dos objetos en el espacio métrico, se necesita contar con un conjunto $(M)$ y una métrica $(D)$ para definir la distancia entre los objetos. Entonces, la congruencia many-to-many sería equivalente a calcular el área no sobrepuesta de las funciones distributivas acumulativas de X e Y para obtener $\bigoplus_{\mathrm{CDF}}$ (Lupu et al., 2017). 
Otra medición de congruencia común es la superposición de la función de densidad (Probability Density Function, posteriormente: PDF) o coeficiente Bhattacharyya (v), que calcula el área de intersección de ambas distribuciones (Andeweg, 2011; Lupu et al., 2017; Selios, 2015). ${ }^{7}$ Esto puede generar una confusión, ya que para $\bigoplus_{\mathrm{CDF}}$, como para las distancias entre medias o estimaciones puntuales, a menor valor existe mayor cercanía; para $\bigoplus_{\mathrm{PDF}}$, en cambio, a mayor valor, mayor congruencia (Lupu et al., 2017; Lupu y Warner, 2017).

$$
\text { (v) Intersección }=\sum_{c=1}^{c} \min \left(\mathrm{j}_{\mathrm{c}} ; \mathrm{l}_{\mathrm{c}}\right)
$$

Finalmente, otra medición reciente que permite superar las limitaciones de las mediciones clásicas de congruencia, es Earth Mover's Distance, posteriormente: EMD, que se deriva del campo de las ciencias de la computación (Rubner et al., 2000).

De acuerdo con Lupu et al. (2017), se puede explicar EMD de la siguiente forma: si se busca medir el autoposicionamiento en una escala de cero a diez, con datos de ubicación y densidad de dos conjuntos $P_{\mathrm{x}}=\{(2,0,5),(5,0,3),(8,0,2)\}$ y $P_{\mathrm{y}}=\{(4,0,3),(5,0,4),(6,0,3)\}, \mathrm{EMD}$ entre $\mathrm{X}$ e $\mathrm{Y}$ es definido por la solución para una optimización lineal del problema, donde el flujo óptimo $f_{\text {ij }}$ para el movimiento de distancia entre ambos conjuntos permite obtener $\bigoplus_{\mathrm{EMD}}$. Así, una definición formal ofrecida por Lupu et al. (2017), una vez encontrado el flujo óptimo, es la siguiente (vi). En el indicador, de forma similar los indicadores de many-to-one, a mayor valor, existe mayor distancia y menor congruencia.

$$
\text { (vi) } \bigoplus_{\text {EMD }}=\frac{\sum_{i=1}^{\mathrm{m}} \sum_{j=1}^{\mathrm{n}} f_{\mathrm{ij}}^{*} \mathrm{~d}_{\mathrm{ij}}}{\sum_{i=1}^{\mathrm{m}} \sum_{j=1}^{\mathrm{n}} f_{\mathrm{ij}}^{*}}
$$

7 Si bien el concepto de intersección es bastante intuitivo, presenta ciertos problemas, tal como indica Selios (2015). Por ejemplo, si toda la élite estuviese en determinado punto y la ciudadanía en el extremo opuesto (e.g. uno y diez), el índice sería cero, al igual que si estuviesen separados por una distancia menor (e.g. cinco y seis). 
Es así como, para el ejemplo indicado, se genera una transferencia de datos entre $P_{\mathrm{x}}$ y $P_{\mathrm{y}}(\mathrm{vii}) .{ }^{8}$ Este ejercicio permite obtener $\bigoplus_{\mathrm{EMD}}$, que para el ejemplo sería $\frac{(1)(1,7)}{1}=1,7$.

(vii) $(|4-2|)(0,3)+(|5-5|)(0,3)+(|5-2|)(0,1)+(|6-2|)(0,1)+(|6-8|)(0,2)=1,7$

La principal ventaja de EMD es que permite estudiar la congruencia de un amplio rango de preferencias y áreas, posibilitando comparaciones de distribuciones con un costo mínimo de procesamiento de datos y gran precisión (Lupu et al., 2017).

\section{Método}

\section{Datos}

Para medir la opinión de la ciudadanía se utilizan las encuestas del proyecto "A Crisis of Legitimacy: Challenges of the Political Order in Argentina, Chile, and Uruguay", financiado por IDRC de Canadá y coordinado por la Universidad Diego Portales, posteriormente: UDP. Estas encuestas fueron realizadas con entrevistas cara a cara en Chile $(N=1.200)$ y Uruguay $(N=1.202)$. El muestreo fue probabilístico en todas las etapas y las encuestas son representativas de la población nacional mayor de 18 años. El nivel de confianza es del $95 \%$ y el error $\pm 2,8 \%$. El trabajo de campo se efectuó en marzo y abril de 2013 en el caso chileno, y en febrero de 2014 en Uruguay. 9

Por otra parte, las preferencias de la élite se miden con encuestas a una muestra de congresistas y miembros del poder ejecutivo realizadas en el marco del mismo proyecto de investigación en Chile $(N=164)$ y Uruguay $(N$ $=121)$, de forma similar a la estrategia metodológica de trabajos como Lupu et al. (2017), Buquet y Selios (2017) y Lupu y Warner (2017). ${ }^{10}$ Para

8 Para más detalles sobre la medición, véase Lupu et al. (2017). Por otra parte, los cálculos se pueden simplificar usando el paquete emdist para R. Precisamente este paquete se utiliza en los análisis de este artículo. Para más información, véase Urbanek y Rubner (2015).

9 Para más detalles metodológicos, véase Joignant et al. (2017). También véanse otros trabajos con estos datos o submuestras enfocados en Argentina (Lupu y Warner, 2017), Chile (Siavelis, 2017) y Uruguay (Buquet y Selios, 2017).

10 Lupu et al. (2017) también identifican otras formas de medir las preferencias de la élite, como los party manifestos (Powell, 2009) o las percepciones de los votantes sobre las plataformas partidarias (Golder y Stramski, 2010). 
los análisis de este trabajo se ocupan las encuestas aplicadas a congresistas solamente, pues son cargos electos democráticamente y no nombramientos basados en confianza política, como es el caso de los gabinetes en los presidencialismos estudiados. ${ }^{11}$

En Chile la tasa de respuesta de los diputados alcanzó 80,73\% $(n=97)$ y $89,47 \%(n=34)$ para senadores. En Uruguay, por otro lado, la tasa de respuesta de los diputados fue $77,78 \%(n=77)$, mientras que en senadores fue de $76,67 \%(n=23)$.

\section{Dimensiones de análisis y medición}

Esta investigación se enfoca en describir los niveles de congruencia éliteciudadanía en Chile y Uruguay, con métodos de medición que permiten una desagregación de la congruencia en tres dimensiones: a) ideológica por bloques políticos; b) temática, apoyo y satisfacción con la democracia; y c) en preferencias sobre el rol del Estado en la economía. Para esto se calculan los niveles de congruencia agregados y se realiza una distinción dependiendo del nivel socioeconómico de la población, para determinar si existen brechas entre los grupos, lo cual permite evaluar la existencia de desigualdad política o sesgo de clase. Las tres dimensiones se analizan en una perspectiva many-tomany con $\bigoplus_{\text {EMD }}$ (Lupu et al., 2017; Rubner et al., 2000). Adicionalmente, la congruencia ideológica también se evalúa con la medida many-to-one RCC de Golder y Stramski (2010), asumiendo como supuesto la existencia de una ciudadanía heterogénea y una élite más bien homogénea.

La dimensión ideológica se operacionaliza con el autoposicionamiento de la élite y de la ciudadanía, usando la escala de medición estándar en encuestas de opinión pública, la cual va de cero a diez. ${ }^{12} \mathrm{La}$ segunda dimensión mide la congruencia temática ${ }^{13}$ que incluye la percepción sobre el voto obligatorio, el apoyo a la democracia ${ }^{14}$ y la satisfacción

11 Esto es una diferencia con Buquet y Selios (2017), quienes usan toda la muestra para sus análisis. Cabe recordar que toda la muestra, como se ha indicado, incluye una selección no probabilística de miembros del poder ejecutivo.

12 Donde cero representa el sector más de izquierda y diez el sector más de derecha.

13 Se utiliza la siguiente pregunta: "Del siguiente listado, ¿cuál cree usted es el principal problema del país?: a) educación; b) delincuencia; c) salud; d) pensiones; e) vivienda; f) obras públicas; g) deterioro del medioambiente; h) transporte público; i) justicia; j) desempleo; k) inflación; 1) política; m) corrupción; n) desigualdad; y o) discriminación”.

14 Se usa la pregunta: “¿Con cuál de las siguientes frases está usted más de acuerdo?: a) la democracia es preferible a cualquier otra forma de gobierno; $b$ ) en algunas circunstancias, un 
con su funcionamiento. ${ }^{15}$ Por último, la tercera dimensión, enfocada en las preferencias sobre el rol del Estado en la economía, utiliza un conjunto de preguntas que miden si el encuestado está de acuerdo o en desacuerdo con las siguientes frases: a) el transporte público debería estar en manos de una empresa estatal; b) debería haber más bancos estatales; c) los colegios particulares-subvencionados deberían pasar al Estado; d) debería existir una red de farmacias estatales; y e) todas las universidades privadas deberían pasar a ser del Estado. ${ }^{16}$

\section{Resultados}

\section{Congruencia y distribución ideológica por bloques politicos}

Una inspección visual permite advertir que la distancia entre la distribución del autoposicionamiento ideológico de la élite y la ciudadanía tiende a ser menor en Chile (Figura 1). ${ }^{17}$ Eso se confirma con el área de intersección de ambas distribuciones: Chile presenta $\bigoplus_{\mathrm{PDF}}=0,810$ y Uruguay $\bigoplus_{\mathrm{PDF}}=0,370$. Sin embargo, hay que considerar que en Chile 38,08\% de la ciudadanía no se autoposiciona en el continuo izquierda-derecha; mientras que en Uruguay la cifra corresponde a 20,71\%. Entre los legisladores las cifras de desafección son bastante bajas: solo $0,77 \%$ en Chile y $2 \%$ en Uruguay. ${ }^{18}$

gobierno autoritario puede ser preferible a uno democrático; y c) a la gente como uno, nos da lo mismo un régimen democrático que uno autoritario".

15 Se mide con una escala Likert de cuatro categorías que van desde "nada satisfecho" a "muy satisfecho".

16 Si bien las encuestas tenían más preguntas de este tipo, se seleccionaron los ítems que permiten comparación. Aun así, el cálculo de congruencia entre la élite y la ciudadanía uruguaya en esta dimensión resulta imposible de realizar, ya que solo se aplicó esta batería de preguntas en la encuesta de élite y no en la encuesta nacional.

17 Las figuras y las tablas se encuentran en el Anexo, al final del presente artículo (Nota del editor).

18 Además, es importante considerar que el autoposicionamiento opera como un concepto grueso que no da cuenta de las complejidades específicas de las significancias de la ideología y lo ideológico. En consecuencia, se encuentra expuesto a problemas de deseabilidad social e imprecisiones de medición. De hecho, en América Latina existen distintos significados, para ciudadanos y para la élite política, asociados al eje ideológico. Estos se componen principalmente de elementos políticos y económicos (Gramacho y Llamazares, 2007; Zechmeister y Corral, 2010). 
Los patrones identificados al evaluar la distribución del autoposicionamiento ideológico se ratifican con los distintos niveles de congruencia, calculados con $\bigoplus_{\text {EMD }}$ y RCC (Tabla 1). Al evaluar los valores de EMD, se advierte que hay mayor congruencia élite-ciudadanía en Chile $\left(\bigoplus_{\mathrm{EMD}}=0,419\right)$ que en Uruguay $\left(\bigoplus_{\mathrm{EMD}}=2,995\right)$. Sin embargo, al considerar los desafectados ideológicos en el cálculo, el escenario tiende a cambiar: en Chile la distancia aumenta 500,24\% $\left(\bigoplus_{\mathrm{EMD}}=2,515\right)$, y en Uruguay solo $25,84 \%$ $\left(\bigoplus_{\mathrm{EMD}}=3,769\right)$. En este sentido, la mayor alineación ideológica entre élite y ciudadanía en Chile no es tan elevada si se considera la desafección política en la medición.

Por otra parte, en Chile la población de menor nivel socioeconómico está más alineada ideológicamente con la élite, aunque en la coalición de centroderecha hay más congruencia con los sectores de nivel socioeconómico más elevado. A pesar de lo anterior, la coalición de centroderecha tiende a estar más alienada con el conjunto de la ciudadanía, pues la coalición de centroizquierda tiene brechas más considerables con los sectores más acomodados de la población. Al evaluar la congruencia ideológica en Chile con RCC, medición que por su cálculo asume la existencia de una ciudadanía heterogénea y una élite más bien homogénea, se aprecia que hay más congruencia entre la élite política y la población de mayor nivel socioeconómico, tanto a nivel agregado como entre los distintos bloques políticos.

En Uruguay, por otra parte, la población de nivel socioeconómico más bajo también tiene mayor congruencia ideológica con la élite. A nivel de bloques políticos, el Frente Amplio mantiene este alineamiento con los sectores menos acomodados, a pesar de que a nivel agregado verifica brechas más amplias con la sociedad en su conjunto; mientras que el Partido Nacional es el más cercano a la ciudadanía en un nivel agregado. Por otra parte, el Partido Nacional y Colorado tienden a tener más congruencia ideológica con la población de mayor nivel socioeconómico. Al evaluar la congruencia ideológica uruguaya con RCC, se tiende a reforzar una alineación con los sectores de menor nivel socioeconómico y la cercanía del Partido Nacional a la ciudadanía de forma transversal.

\section{Congruencia temática, apoyo y satisfacción con la democracia}

El apoyo a la democracia es mucho más fuerte en la élite que en la ciudadanía. En Uruguay, todos los diputados y senadores encuestados prefieren la democracia como forma de gobierno; en tanto, en Chile alcanzan el 96,18\%. Entre la ciudadanía, las cifras son más bajas. En Chile, la preferencia por la 
democracia alcanza 54,94\% y en Uruguay, 73,80\%. Los niveles de congruencia para apoyo a la democracia muestran que hay mayor congruencia éliteciudadanía en Uruguay. Por otra parte, en ambos países, los sectores más acomodados son los que tienden a apoyar más la democracia (Tabla 2). Esto implica que los sectores menos aventajados podrían eventualmente alinearse con cierta facilidad con posturas no democráticas.

A pesar de lo anterior, la situación se invierte al evaluar la satisfacción con la democracia: en Chile hay menos brechas al respecto. Esto es porque la élite chilena es bastante autocomplaciente: $74,05 \%$ está satisfecho o muy satisfecho con el funcionamiento de la democracia; mientras que en Uruguay, el porcentaje solo alcanza 7\%. Pese a que en Chile solo 48,16\% de la población está satisfecho o muy satisfecho con la democracia, mientras que en Uruguay la cifra alcanza $72,69 \%$, la congruencia es mayor en Chile.

En el caso del voto obligatorio, solo $29,91 \%$ de la población chilena lo apoya, mientras que en Uruguay, $53,47 \%$. Entre la élite el apoyo es más alto: $64,89 \%$ en Chile y $88 \%$ en Uruguay. La congruencia sobre este tema es similar en ambos países, siendo un poco mayor con la población de mayor nivel socioeconómico. Por último, la congruencia sobre los problemas públicos relevantes es similar en ambos países, aunque se enfoca en temas diferentes (Figura 2). Por otro lado, se advierte una brecha importante por nivel socioeconómico: hay mayor congruencia entre la élite y los sectores más acomodados de la población. Esto podría considerarse como un alineamiento asociado con un fortalecimiento de la opinión y la voz política de los individuos con mayores recursos sociales y económicos, rasgo de desigualdad política.

\section{Congruencia en preferencias sobre el rol del Estado}

Las preferencias por más intervención estatal son llamativas en Chile, ya que durante la dictadura se implementaron con bastante éxito reformas estructurales que implicaron una importante reducción del Estado y la creación de mercados no convencionales, con bajos niveles de regulación e intervención estatal (Solimano, 2014). Este modelo económico no se alteró con la recuperación democrática, al contrario, una serie de elementos garantizaron su intocabilidad (Garretón, 1999; Huneeus, 2014).

Las cifras son particularmente altas en temas relacionados con mayor intervención en mercados que han tenidos problemas de colusión, actividades monopólicas y tráfico de influencias en los últimos años. Por ejemplo, $79,57 \%$ de los chilenos se muestra de acuerdo con la creación de una red de 
farmacias estatales, de forma similar 75,29\% piensa que deberían crearse más bancos estatales. También existe una alta preferencia, con $63,66 \%$, por la estatización del transporte público urbano, lo cual resulta llamativo considerando que es una de las áreas más criticadas en términos de implementación de política pública en Chile. De igual forma es llamativa la idea de que las universidades privadas y los colegios particulares-subvencionados debiesen pasar al control del Estado (59,57\% y 53,7\%, respectivamente).

En este contexto, se verifican algunas brechas evidentes entre la ciudadanía y la élite política en algunas de estas áreas. ${ }^{19}$ La élite política se muestra bastante de acuerdo con implementar una red de farmacias estatales en Chile (59,54\%), no así en Uruguay (37\%). Por otra parte, en el resto de los temas hay diferencias más relevantes: en Chile solo 44,28\% de la élite se muestra de acuerdo con la creación de más bancos estatales, mientras que en Uruguay la cifra es de 33\%. Respecto a la estatización del transporte en Chile, 48,09\% está de acuerdo, y en Uruguay solo $22 \%$.

Por último, las brechas relevantes se perciben en torno al manejo de la educación secundaria y superior. En Chile, solo 9,16\% está de acuerdo con que las universidades privadas pasen al control del Estado, cifra cercana a Uruguay (10\%); respecto al control estatal de los colegios particularessubvencionados, la cifra se mantiene en Chile (9,92\%) y es levemente superior en Uruguay (16\%).

$\mathrm{Al}$ evaluar la congruencia con $\bigoplus_{\mathrm{EMD}}$ en Chile (Tabla 3), se advierte que las principales brechas corresponden a temas educacionales, donde además se observan brechas por nivel socioeconómico: la población más acomodada tiende a estar más de acuerdo con la élite política, la cual se resiste al manejo estatal de colegios y universidades. Esto se alinea con los recientes cuestionamientos a los pilares del modelo chileno, tales como la Constitución y los sistemas educacional, tributario y previsional (Cortés, 2015). ${ }^{20}$

19 Para Uruguay solo están disponibles los datos de la encuesta de élite, ya que, como se indicó previamente, no se aplicaron estas preguntas en la encuesta nacional.

20 En este contexto, se ha instalado un debate sobre un posible derrumbe del modelo económico chileno (Mayol, 2012). Sin embargo, dicho debate es más bien local y posee un carácter más coyuntural que académico. Para una reflexión teórica sobre variedades de neoliberalismo y el modelo chileno, véase Maillet (2015a y 2015b). 


\section{Conclusiones}

Este artículo ofrece un diagnóstico sobre las preferencias de la élite política y la ciudadanía en Chile y Uruguay. Para esto se trabaja con encuestas de opinión nacionales y de élites, las cuales permiten evaluar tres dimensiones de congruencia: a) ideológica; b) temática, de apoyo y satisfacción con la democracia; y c) en preferencias sobre el rol del Estado en la economía. Se utilizan principalmente medidas many-to-many e índices de congruencia recientes que permiten mayor precisión en el diagnóstico de las brechas y alineamientos existentes.

En la primera dimensión, se advierte una mayor congruencia en Chile que en Uruguay, aunque la diferencia entre ambos países tiende a acercarse si se consideran los desafectados ideológicos en el cálculo, los cuales corresponden a 38,08\% en Chile. Las mediciones con EMD permiten apreciar que en Chile la coalición de centroderecha tiende a estar más alineada con la ciudadanía; mientras que en Uruguay se advierte que el Frente Amplio tiene brechas relevantes con la población. Por otro lado, al utilizar RCC, se advierte que la élite, al considerarse como cuerpo homogéneo, tiende a alinearse ideológicamente con la población de mayor nivel socioeconómico en el caso chileno y con los sectores más desaventajados en Uruguay.

En la segunda dimensión, se percibe una alta congruencia élite-ciudadanía en el apoyo a la democracia en Uruguay, no así en la satisfacción con esta. Esto no es porque en Chile exista la percepción de que la democracia funciona mejor, sino porque la élite uruguaya es bastante autocrítica con su propia democracia. Respecto a la congruencia sobre problemas públicos relevantes y voto obligatorio, se observa que hay mayor congruencia entre la élite y los sectores de mayor nivel socioeconómico de la población.

Por último, en torno a la congruencia sobre preferencias del rol del Estado en la economía, se advierte una predisposición de la ciudadanía chilena hacia una mayor regulación e intervención estatal. Dichas tendencias son más conservadoras en la élite chilena y uruguaya. Las principales brechas en esta materia se aprecian en el manejo educacional en Chile: mientras la ciudadanía muestra tendencias hacia la estatización de universidades y colegios, la élite política se resiste a ello. Esto es consistente con las recientes movilizaciones sociales y el posicionamiento de la educación como un problema relevante en la agenda pública chilena.

Si bien todos estos resultados ofrecen un diagnóstico sobre las distintas brechas que existen entre la ciudadanía y la élite en dos democracias consolidadas en América Latina, es importante considerar que dichos resultados 
deben tener en cuenta posibles recambios recientes en las élites gobernantes y, además, deben ser contrastados con datos longitudinales, para delinear un diagnóstico más robusto. De todas maneras, este trabajo ofrece resultados con tendencias que resultan llamativas y requieren de mayor estudio, además presenta un estado del arte actualizado con una aplicación práctica sobre mediciones de congruencia política y su vínculo con la teoría de élites.

\section{Referencias}

Achen, Christopher (1977), "Measuring Representation: Perils of the Correlation Coefficient", en American Journal of Political Science, vol. 21, núm. 4, Estados Unidos: Midwest Political Science Association. DOI: 10.2307/2110737. Disponible en: http://www.jstor.org/stable/2110737 [28 de febrero de 2018].

Achen, Christopher (1978), "Measuring Representation", en American Journal of Political Science, vol. 22, núm. 3, Estados Unidos: Midwest Political Science Association. DOI: 10.2307/2110458. Disponible en: http://www.jstor.org/stable/2110458 [28 de febrero de 2018].

Alcántara, Manuel (2013), "De políticos y política: profesionalización y calidad en el ejercicio público", en Perfiles Latinoamericanos, vol. 21, núm. 41, México: Facultad Latinoamericana de Ciencias Sociales. Disponible en: http://ref.scielo.org/ff37bw [19 de abril de 2017].

Alcántara, Manuel y Luna, Juan Pablo (2004), "Ideología y competencia partidaria en dos post-transiciones: Chile y Uruguay en perspectiva comparada", en Revista de Ciencia Política, vol. 24, núm. 1, Chile: Pontificia Universidad Católica de Chile. DOI: 10.4067/S0718-090X2004000100006. Disponible en: http://dx.doi.org/10.4067/ S0718-090X2004000100006 [28 de febrero de 2018].

Andeweg, Ruby (2011), "Approaching Perfect Policy Congruence. Measurement, Development, and Relevance for Political Representation", en Rosema, Martin et al. [comps.], How Democracy Works. Political Representation and Policy Congruence in Modern Societies, Países Bajos: Amsterdam University Press.

Andeweg, Rudy y Thomassen, Jacques (2005), "Modes of Political Representation: Toward a New Typology", en Legislative Studies Quarterly, vol. 30, núm. 4, Estados Unidos: American Political Science Association. DOI: 10.3162/036298005X201653. Disponible en: http://doi.org/10.3162/036298005X201653 [20 de abril de 2018].

Arana, Ignacio (2016), "¿Cómo evaluar a los integrantes de la élite política? Una propuesta basada en los presidentes americanos”, en Politica, Revista de Ciencia Politica, vol. 54, núm. 1, Chile: Universidad de Chile. Disponible en: http://revistapolitica.uchile.cl/ index.php/RP/article/view/42714 [28 de febrero de 2018].

Barreda, Mikel y Ruiz, Leticia (2015), "Contrapuntos en torno al modo de representación: ciudadanos y diputados de América Latina”, ponencia presentada en el VIII Congreso Latinoamericano de Ciencia Politica, 22-24 julio, Lima, Perú (inédito).

Bellinger, Paul y Arce, Moisés (2011), "Protest and Democracy in Latin America's Market Era”, en Political Research Quarterly, vol. 64, núm. 3, Estados Unidos: Western Political Science Association. Disponible en: http://www.jstor.org/stable/23056385 [21 de abril de 2018]. 
Best, Heinrich (2011), "The Elite-Population Gap in the Formation of Political Identities. A Cross-Cultural Investigation”, en Europe-Asia Studies, vol. 63, núm. 6, Escocia: Central and East European Studies. DOI: 10.1080/09668136.2011.585751. Disponible en: http://doi.org/10.1080/09668136.2011.585751 [28 de febrero de 2018].

Bobbio, Norberto (2003), "Élites, teoría delle", en Bobbio, Norberto et al. [comps.], Dizionario di Politica, Italia: Unione Tipografico-Editrice Torinese.

Buquet, Daniel y Selios, Lucía (2017), "Political Congruence in Uruguay, 2014”, en Joignant, Alfredo et al. [comps.], Malaise in Representation in Latin American Countries, Estados Unidos: Palgrave Macmillan.

Cantillana, Carlos et al. (2017), "Malestar con la representación democrática en América Latina”, en Política y Gobierno, vol. XXIV, núm. 2, México: Centro de Investigación y Docencia Económicas. Disponible en: http://ref.scielo.org/m2vz3z [20 de abril de 2018].

Codato, Adriano y Espinoza, Fran (2018), Élites en las Américas: Diferentes Perspectivas, Brasil: Editora Universidade Federal do Paraná.

Cortés, Alexis (2015): “Chile en la encrucijada de un nuevo ciclo", en Cadernos de Trabalho NETSAL, vol. 3, núm. 7, Brasil: Universidade do Estado do Rio de Janeiro. Disponible en: http://www.academia.edu/31489911/Chile_en_la_encrucijada_de_un_nuevo_ ciclo [07 de noviembre de 2018].

Dalton, Russell et al. (2010), “The Individual-Institutional Nexus of Protest Behaviour”, en British Journal of Political Science, vol. 40, núm. 1, Inglaterra: Cambridge University Press. DOI: 10.1017/S000712340999038X. Disponible en: http://doi.org/10.1017/ S000712340999038X [28 de febrero de 2018].

Dalton, Russell et al. (2011), “The Dynamics of Political Representation”, en Rosema, Martin et al. [comps.], How Democracy Works. Political Representation and Policy Congruence in Modern Societies, Países Bajos: Amsterdam University Press.

Dahl, Robert (1961), Who Governs? Democracy and Power in an American City, Estados Unidos: Yale University Press.

Dubrow, Joshua (2008), "Guest Editor's Introduction: Defining Political Inequality within a Cross-National Perspective”, en International Journal of Sociology, vol. 37, núm. 4, Estados Unidos: Taylor \& Francis. Disponible en: http://www.jstor.org/ stable/20628308 [28 de febrero de 2018].

Garretón, Manuel Antonio (1999), "Chile 1997-1998: The Revenge of Incomplete Democratization”, en International Affairs, vol. 75, núm. 2, Inglaterra: Royal Institute of International Affairs. Disponible en: https://www.jstor.org/stable/2623343 [07 de noviembre de 2018].

Golder, Matt y Stramski, Jacek (2010), "Ideological Congruence and Electoral Institutions”, en American Journal of Political Science, vol. 54, núm. 1, Estados Unidos: Midwest Political Science Association. DOI: 10.1111/j.1540-5907.2009.00420.x. Disponible en: http://doi.org/10.1111/j.1540-5907.2009.00420.x [28 de febrero de 2018].

González-Bustamante, Bastián (2016), "Élites políticas, económicas e intelectuales: una agenda de investigación creciente para la ciencia política”, en Politica, Revista de Ciencia Política, vol. 54, núm. 1, Chile: Universidad de Chile. Disponible en: http:// revistapolitica.uchile.cl/index.php/RP/article/view/42690 [28 de febrero de 2018].

González-Bustamante, Bastián y Garrido-Vergara, Luis (2018), "Socialización, trayectorias y poscarrera de ministros en Chile, 1990-2010”, en Politica y Gobierno, vol. XXV, núm. 1, México: Centro de Investigación y Docencia Económicas. Disponible en: http:// ref.scielo.org/bm9fmn [20 de abril de 2018]. 
González-Bustamante, Bastián y Olivares, Alejandro (2018), "La élite política gubernamental en Chile: supervivencia de ministros", en Codato, Adriano y Espinoza, Fran [comps.], Élites en las Américas: Diferentes Perspectivas, Brasil: Editora Universidade Federal do Paraná.

Gramacho, Wladimir y Llamazares, Iván (2007), "Ideología y preferencias de intervención económica estatal entre los diputados latinoamericanos”, en Documentos CIDOB, núm. 18, España: Barcelona Centre for International Affairs. Disponible en: http://americo. usal.es/OIR/OPAL/pdfs/Gramacho_Llamazares.pdf [22 de abril de 2018].

Gulbrandsen, Trygve y Engelstad, Fredrik (2005), "Elite consensus on the Norwegian welfare state model”, en West European Politics, vol. 28, núm. 4, Inglaterra: Routledge. DOI: 10.1080/01402380500217029. Disponible en: http://doi. org/10.1080/01402380500217029 [28 de febrero de 2018].

Higley, John y Gunther, Richard (1992), Elites and Democratic Consolidation in Latin America and Southern Europe, Inglaterra: Cambridge University Press.

Huneeus, Carlos (2014), La democracia semisoberana. Chile después de Pinochet, Chile: Taurus.

Joignant, Alfredo (2011), "Tecnócratas, technopols y dirigentes de partido: tipos de agentes y especies de capital en las elites gubernamentales de la Concertación (1990-2010)”, en Joignant, Alfredo y Güell, Pedro [comps.], Notables, tecnócratas y mandarines: Elementos de sociología de las elites en Chile (1990-2010), Chile: Ediciones Universidad Diego Portales.

Joignant, Alfredo et al. (2017), Malaise in Representation in Latin American Countries, Estados Unidos: Palgrave Macmillan.

Kitschelt, Herbert (2000), "Linkages between Citizens and Politicians in Democratic Polities”, en Comparative Political Studies, vol. 33, núm. 6-7, Estados Unidos: Sage. DOI: 10.1177/001041400003300607. Disponible en: http://journals.sagepub.com/ doi/abs/10.1177/001041400003300607 [20 de abril de 2018].

Kitschelt, Herbert et al. (2010), Latin American Party Systems, Estados Unidos: Cambridge University Press.

Levine, Daniel y Molina, José (2011), The Quality of Democracy in Latin America, Estados Unidos: Lynne Rienner Publishers.

Lipset, Seymour (1960), Political Man: The Social Bases of Politics, Estados Unidos: Doubleday \& Company.

Luna, Juan PabloyZechmeister, Elizabeth (2005), "Political Representation in Latin America: A Study of Elite-Mass Congruence in Nine Countries", en Comparative Political Studies, vol. 38, núm. 4, Estados Unidos: Sage. DOI: 10.1177/0010414004273205. Disponible en: http://journals.sagepub.com/doi/abs/10.1177/0010414004273205 [28 de febrero de 2018].

Luna, Juan Pablo y Zechmeister, Elizabeth (2010), "Political Representation in Latin America”, en Kitschelt, Herbert et al. [comps.], Latin American Party Systems, Estados Unidos: Cambridge University Press.

Lupu, Noam et al. (2017), "A New Measure of Congruence: The Earth Mover's Distance”, en Political Analysis, vol. 25, núm. 1, Estados Unidos: PolMeth. DOI: 10.1017/ pan.2017.2. Disponible en: http://doi.org/10.1017/pan.2017.2 [20 de abril de 2018].

Lupu, Noam y Warner, Zach (2017), "Mass-Elite Congruence and Representation in Argentina”, en Joignant, Alfredo et al. [comps.], Malaise in Representation in Latin American Countries, Estados Unidos: Palgrave Macmillan.

Mainwaring, Scott (2006), "The Crisis of representation in the Andes", en Journal of Democracy, vol. 17, núm. 3, Estados Unidos: National Endowment for Democracy. DOI: $10.1353 /$ jod.2006.0048. Disponible en: http://muse.jhu.edu/article/200110 [28 de febrero de 2018]. 
Maillet, Antoine (2015a), “Más allá del 'modelo' chileno: una aproximación multi-sectorial a las relaciones Estado-mercado", en Revista de Sociologia e Politica, vol. 23, núm. 55, Brasil: Universidade Federal do Paraná. DOI: 10.1590/1678-987315235504. Disponible en: http://ref.scielo.org/xcpns9 [07 de noviembre de 2018].

Maillet, Antoine (2015b), "Variedades de Neoliberalismo. Innovación conceptual para el análisis del rol del Estado en los mercados", en Revista de Estudios Políticos (Nueva Época), núm. 169, España: Centro de Estudios Políticos y Constitucionales. DOI: 10.18042/cepc/rep.169.04. Disponible en: http://doi.org/10.18042/cepc/rep.169.04 [07 de noviembre de 2018].

Marshall, Thomas H. (1950), Citizenship and Social Class and Other Essays, Inglaterra: Cambridge University Press.

Mayol, Alberto (2012), El derrumbe del modelo. La crisis de la economía de mercado en el Chile contemporáneo, Chile: LOM.

Miller, Warren y Stokes, Donald (1963), “Constituency Influence in Congress", en American Political Science Review, vol. 57, núm. 1, Estados Unidos: American Political Science Association. DOI: 10.2307/1952717. Disponible en: http://www.jstor.org/ stable/1952717 [20 de abril de 2018].

Mills, C. Wright (1956), The Power Elite, Estados Unidos: Oxford University Press.

Morales, Mauricio (2014), "Congruencia programática entre partidos y votantes en Chile", en Perfiles Latinoamericanos, vol. 22, núm. 44, México: Facultad Latinoamericana de Ciencias Sociales. Disponible en: http://ref.scielo.org/wh3g9m [28 de febrero de 2018].

Otero-Felipe, Patricia (2014), "Party-voter linkages. A Comparative View of Ideology and European Integration”, en Revista Española de Investigaciones Sociológicas, núm. 146, España: Centro de Investigaciones Sociológicas. DOI: 10.5477/cis/reis.146.141. Disponible en: http://doi.org/10.5477/cis/reis.146.141 [28 de febrero de 2018].

Pakulski, Jan (2012), "Introduction: John Higley's Work on Elite Foundations of Social Theory and Politics", en Historical Social Research-Historische Sozialfourschung, vol. 37, núm. 1, Alemania: Leibniz Institute for the Social Sciences. Disponible en: http:// www.jstor.org/stable/41756447 [28 de febrero de 2018].

Parry, Geraint (2005), Political Elites, Inglaterra: European Consortium for Political Research Press.

Pasquino, Gianfranco (2011), Nuevo curso de ciencia politica, México: Fondo de Cultura Económica.

Pitkin, Hanna (1967), The Concept of Representation, Estados Unidos: University of California Press.

Powell, G. Bingham (2004), "Political Representation in Comparative Politics", en Annual Review of Political Science, vol. 7, Estados Unidos: Annual Reviews. DOI: 10.1146/ annurev.polisci.7.012003.104815. Disponible en: http://doi.org/10.1146/annurev. polisci.7.012003.104815 [20 de abril de 2018].

Powell, G. Bingham (2009), "The Ideological Congruence Controversy. The Impact of Alternative Measures, Data, and Time Periods on the Effects of Election Rules", en Comparative Political Studies, vol. 42, núm. 12, Estados Unidos: Sage. DOI: 10.1177/0010414009332147. Disponible en: http://journals.sagepub.com/ doi/10.1177/0010414009332147 [22 de abril de 2018].

Programa de las Naciones Unidas para el Desarrollo (PNUD) (2014), Ciudadanía Política: Voz y Participación Ciudadana en América Latina, Argentina: Siglo XXI. 
Przeworski, Adam et al. (1999), Democracy, Accountability, and Representation, Inglaterra: Cambridge University Press.

Real-Dato, José (2017), "Party-voters Congruence Concerning the European Union: An Analysis of Its Evolution During the Eurozone Crisis Using Elite and Mass Surveys", en Corvinus Journal of Sociology and Social Policy, vol. 8, núm. 3, Hungría: Corvinus University of Budapest. DOI: 10.14267/cjssp.2017.3S.05. Disponible en: http:// cjssp.uni-corvinus.hu/index.php/cjssp/article/view/268 [20 de abril de 2018].

Rivas, Cristina et al. (2014), “CCómo medir la congruencia ideológica? Elite parlamentaria y opinión pública en América Latina”, en Seminario de Investigación Instituto de Iberoamérica, núm. 9, España: Universidad de Salamanca. Disponible en: http:// americo.usal.es/iberoame/?q=node/2351 [06 de septiembre de 2015].

Rubner, Yossi et al. (2000), “The Earth Mover's Distance as a Metric for Image Retrieval”, en International Journal of Computer Vision, vol. 40, núm. 2, Estados Unidos: Springer. DOI: 10.1023/A:1026543900054. Disponible en: http://link.springer.com/ article/10.1023/A:1026543900054 [19 de abril de 2018].

Sartori, Giovanni (1999), Elementos de teoría politica, España: Alianza.

Selios, Lucía (2015), "Receptividad democrática en América Latina: un análisis de congruencia ideológica en perspectiva diacrónica”, ponencia presentada en el VIII Congreso Latinoamericano de Ciencia Política, 22-24 julio, Lima, Perú (inédito).

Serna, Miguel (2006), "Las vías hacia el poder político. Bases sociales y carreras parlamentarias", en Mazzei, Enrique [comp.], El Uruguay desde la Sociología IV, Uruguay: Universidad de la República.

Siavelis, Peter (2017), "Elite-Mass Congruence in Chile", en Joignant, Alfredo et al. [comps.], Malaise in Representation in Latin American Countries, Estados Unidos: Palgrave Macmillan.

Solimano, Andrés (2014), Economic Elites, Crises, and Democracy: Alternatives beyond Neoliberal Capitalism, Estados Unidos: Oxford University Press.

Torcal, Mariano (2006), "Political Disaffection and Democratization History in New Democracies”, en Torcal, Mariano y Montero, José Ramón [comps.], Political Disaffection in Contemporary Democracies: Social Capital, Institutions, and Politics, Estados Unidos: Routledge.

Urbanek, Simon y Rubner, Yossi (2015), "Earth Mover's Distance. Package providing calculation of Earth Mover's Distance (EMD)". Disponible en: http://cran.r-project. org/web/packages/emdist/emdist.pdf [19 de abril de 2018].

Vommaro, Gabriel y Gené, Mariana (2018), Las élites politicas en el Sur. Un estado de la cuestión de los estudios sobre la Argentina, Brasil y Chile, Argentina: Ediciones Universidad Nacional de General Sarmiento.

Weissberg, Robert (1978), “Collective vs. Dyadic Representation in Congress”, en American Political Science Review, vol. 72, núm. 2, Estados Unidos: American Political Science Association. DOI: 10.2307/1954109. Disponible en: http://www.jstor.org/ stable/1954109 [28 de febrero de 2018].

Zechmeister, Elizabeth y Corral, Margarita (2010), “El variado significado de 'izquierda' y 'derecha' en América Latina”, en Perspectivas desde el Barómetro de las Américas, núm. 38, Estados Unidos: Vanderbilt University. Disponible en: http://www.vanderbilt. edu/lapop/insights/I0838es.pdf [22 de abril de 2018]. 


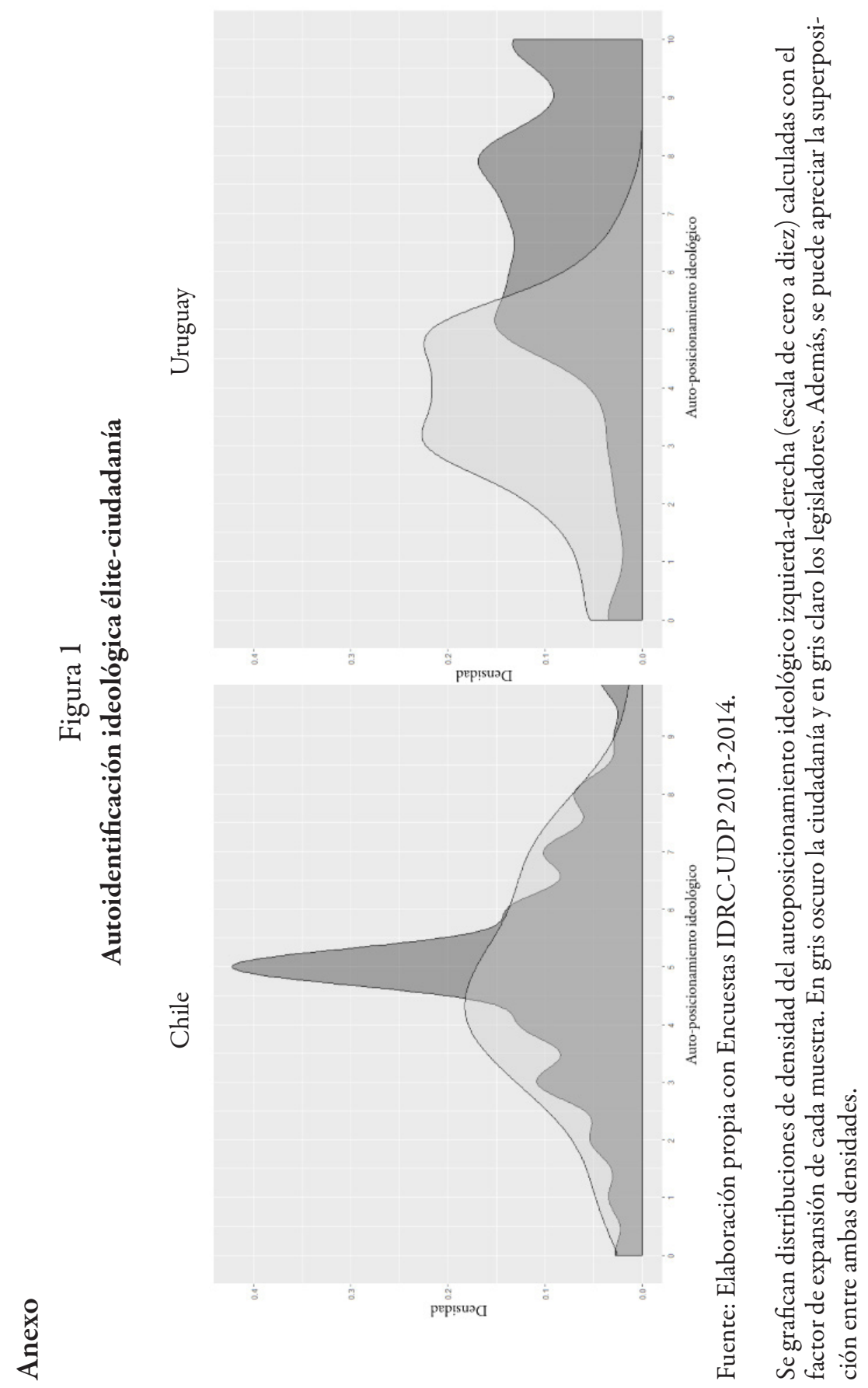




\section{Tabla 1}

\section{Congruencia ideológica por bloque político y nivel socioeconómico}

\begin{tabular}{|c|c|c|c|c|c|c|}
\hline & \multicolumn{3}{|c|}{ Chile } & \multicolumn{3}{|c|}{ Uruguay } \\
\hline & NSE- & NSE+ & Todos & NSE- & NSE+ & Todos \\
\hline Nueva Mayoría & $\begin{array}{l}1,675 \\
(0,363)\end{array}$ & $\begin{array}{l}2,020 \\
(0,320)\end{array}$ & $\begin{array}{l}1,793 \\
(0,355)\end{array}$ & -- & -- & -- \\
\hline Alianza & $\begin{array}{l}1,732 \\
(0,338)\end{array}$ & $\begin{array}{l}1,416 \\
(0,293)\end{array}$ & $\begin{array}{l}1,608 \\
(0,330)\end{array}$ & -- & -- & -- \\
\hline Frente Amplio & -- & -- & -- & $\begin{array}{l}3,461 \\
(0,498)\end{array}$ & $\begin{array}{l}4,140 \\
(0,512)\end{array}$ & $\begin{array}{l}3,932 \\
(0,508)\end{array}$ \\
\hline Partido Nacional & -- & - & -- & $\begin{array}{l}2,157 \\
(0,133)\end{array}$ & $\begin{array}{l}1,990 \\
(0,157)\end{array}$ & $\begin{array}{l}1,998 \\
(0,149)\end{array}$ \\
\hline Partido Colorado & -- & - & -- & $\begin{array}{l}2,513 \\
(0,233)\end{array}$ & $\begin{array}{l}2,436 \\
(0,253)\end{array}$ & $\begin{array}{l}2,444 \\
(0,247)\end{array}$ \\
\hline Todos & $\begin{array}{l}0,388 \\
(0,051)\end{array}$ & $\begin{array}{l}0,539 \\
(0,013)\end{array}$ & $\begin{array}{l}0,419 \\
(0,040)\end{array}$ & $\begin{array}{l}2,575 \\
(0,392)\end{array}$ & $\begin{array}{l}3,203 \\
(0,409)\end{array}$ & $\begin{array}{l}2,995 \\
(0,404)\end{array}$ \\
\hline
\end{tabular}

Fuente: Elaboración propia con Encuestas IDRC-UDP 2013-2014.

Los valores corresponden a $\bigoplus_{\mathrm{EMD}} \mathrm{y}$ entre paréntesis RCC. 


\section{Tabla 2}

\section{Congruencia temática, apoyo y satisfacción con la democracia por nivel socioeconómico}

\begin{tabular}{|c|c|c|c|c|c|c|}
\hline & \multicolumn{3}{|c|}{ Chile } & \multicolumn{3}{|c|}{ Uruguay } \\
\hline & NSE- & NSE+ & Todos & NSE- & NSE+ & Todos \\
\hline $\begin{array}{l}\text { Apoyo a la } \\
\text { democracia }\end{array}$ & 0,814 & 0,388 & 0,658 & 0,701 & 0,332 & 0,485 \\
\hline $\begin{array}{l}\text { Satisfacción con la } \\
\text { democracia }\end{array}$ & 0,836 & 0,403 & 0,637 & 1,229 & 1,485 & 1,410 \\
\hline Voto obligatorio & 0,409 & 0,324 & 0,379 & 0,463 & 0,322 & 0,348 \\
\hline $\begin{array}{l}\text { Problema público más } \\
\text { relevante }\end{array}$ & 1,503 & 0,953 & 1,302 & 1,800 & 0,926 & 1,243 \\
\hline
\end{tabular}

Fuente: Elaboración propia con Encuestas IDRC-UDP 2013-2014.

Los valores corresponden a $\bigoplus_{\mathrm{EMD}}$. 


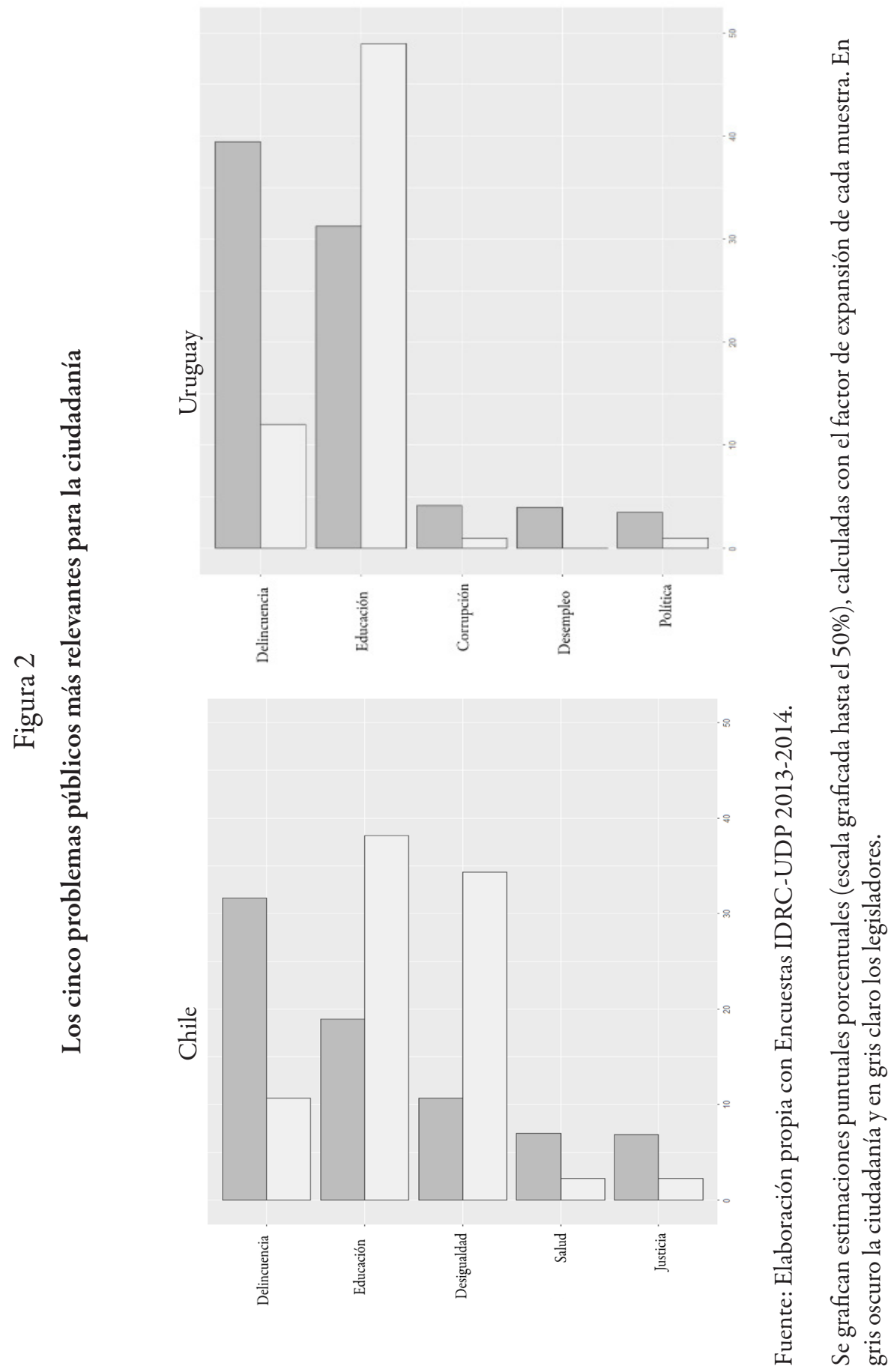




\section{Tabla 3}

Congruencia en preferencias sobre el rol del Estado en la economía

\begin{tabular}{llcc}
\hline & \multicolumn{3}{c}{ Chile } \\
\cline { 3 - 4 } & NSE- & NSE+ & Todos \\
\hline $\begin{array}{l}\text { El transporte público debería estar en } \\
\text { manos de una empresa estatal }\end{array}$ & 0,221 & 0,199 & 0,226 \\
$\begin{array}{l}\text { Debería haber más bancos estatales } \\
\begin{array}{l}\text { Los colegios particulares- } \\
\text { subvencionados deberían pasar al }\end{array}\end{array}$ & 0,362 & 0,347 & 0,359 \\
$\begin{array}{l}\text { Estado } \\
\begin{array}{l}\text { Debería existir una red de farmacias } \\
\text { estatales }\end{array}\end{array}$ & 0,531 & 0,391 & 0,486 \\
$\begin{array}{l}\text { Todas las universidades privadas } \\
\text { deberían pasar a ser del Estado }\end{array}$ & 0,618 & 0,458 & 0,236 \\
\hline
\end{tabular}

Fuente: Elaboración propia con Encuestas IDRC-UDP 2013-2014.

Los valores corresponden a $\bigoplus_{\mathrm{EMD}}$. 
Bastián González-Bustamante. Profesor Instructor del Departamento de Gestión y Políticas Públicas de la Facultad de Administración y Economía de la Universidad de Santiago de Chile. Magíster en Ciencia Política, administrador público y Licenciado en Ciencias Políticas y Gubernamentales por la Universidad de Chile. Principales líneas de investigación: composición y rotación de gabinetes, élites políticas, sistemas de servicio civil, participación política, medios digitales, gobierno electrónico y metodología de la investigación, con foco en métodos cuantitativos y mixtos. Publicaciones recientes: González-Bustamante, Bastián y Garrido-Vergara, Luis (2018), "Socialización, trayectorias y poscarrera de ministros en Chile, 1990-2010", en Politica y Gobierno, vol. XXV, núm. 1, México: Centro de Investigación y Docencia Económicas; González-Bustamante, Bastián (2018), "Civil Service Models in Latin America", en Farazmand, Ali [comp.], Global Encyclopedia of Public Administration, Public Policy, and Governance, Estados Unidos: Springer; González-Bustamante, Bastián (2018), "Internet, uso de redes sociales digitales y participación en el Cono Sur", en Cottet, Pablo [comp.], Opinión Pública Contemporánea: Otras Posibilidades de Comprensión e Investigación, Chile: Social-Ediciones. 